\title{
Modeling dust in a universe of galaxies
}

\author{
Desika Narayanan ${ }^{1,2,3}$ (D), Qi Li ${ }^{1}$, Romeel Davé ${ }^{4,5,6}$, Charlie Conroy ${ }^{7}$, \\ Benjamin D. Johnson ${ }^{7}$ and Gergo Popping ${ }^{8}$ \\ ${ }^{1}$ Department of Astronomy, University of Florida, 211 Bryant Space Sciences Center, \\ Gainesville, FL, 32611, USA \\ email: desika.narayanan@ufl.edu \\ ${ }^{2}$ University of Florida Informatics Institute, 432 Newell Drive, CISE Bldg E251, \\ Gainesville, FL, 32611, USA \\ ${ }^{3}$ Cosmic Dawn Centre, Niels Bohr Institute, University of Copenhagen and DTU-Space, \\ Technical University of Denmark \\ ${ }^{4}$ Institute for Astronomy, Royal Observatory, University of Edinburgh, \\ Edinburgh, EH9 3HJ, UK \\ ${ }^{5}$ University of the Western Cape, Bellville, Cape Town, 7535, South Africa \\ ${ }^{6}$ South African Astronomical Observatories, Observatory, Cape Town, 7925, South Africa \\ ${ }^{7}$ Department of Astronomy, Harvard University, 60 Garden Street, Cambridge, \\ MA, 02138, USA \\ ${ }^{8}$ European Southern Observatory, Karl-Schartzchild-Strasse 2, 85748, \\ Garching, Germany
}

\begin{abstract}
In this invited talk, we discuss the physics of the lifecycle of dust in the context of galaxy formation simulations. After outlining the basic physical processes, we apply algorithms for the formation, growth, and destruction of dust in the ISM to a state-of-the-art cosmological simulation to develop a model for the evolution of the dust to gas and dust to metals ratios in galaxies. We show that while modern simulations are able to match the observed dust mass function at redshift $z=0$, most models underpredict the observed mass function at high-redshift $(z=2)$. We then show the power of these techniques by expanding our model to include a spectrum of dust sizes, and make initial predictions for extinction laws in local galaxies.
\end{abstract}

Keywords. ISM: dust, extinction, galaxies: ISM, galaxies: formation

\section{Introduction}

Dust plays a critical role in the physics of the interstellar medium (ISM) of galaxies, impacting both the chemistry of the ISM via serving as catalysts for molecular hydrogen $\left(\mathrm{H}_{2}\right)$ formation, as well serving as a sink/source of metals as grains are formed/destroyed (Draine (2011)). Similarly, dust impacts the thermal structure of the ISM by acting as an ISM coolant, as well as a shield from far ultraviolet (FUV) radiation (Goldsmith (2001); Draine (2003); Narayanan \& Krumholz (2017)).

And yet, to date, most simulations of galaxy formation and evolution have not explicity included the physics of dust formation, growth, and destruction in the interstellar medium. Typically, dust is included in post-processing, via a simple constant dust mass to metal mass ratio, i.e. akin to $M_{\text {dust }}=0.4 \times M_{\text {metals }}$ (e.g. Hayward et al. (2013); Narayanan et al. (2015, 2018); Abruzzo et al. (2018)), while some slightly more sophisticated parameterizations have included dust-to-metals or dust-to-gas ratios that are a function of the metallicity (e.g. Privon et al. (2018); Lagos et al. (2019)), that derive from observational scaling relations (Rémy-Ruyer et al. (2014); De Vis et al. (2019)). 
The impact of including dust only in post-processing can be significant. First, by basing the dust content on linear scalings with metallicity that are derived from only a few galaxies (e.g. Dwek (1998); Watson (2011)), it is entirely possible that the dust content could be grossly miscalculated for galaxies that have drastically different physical properties (i.e. locations in metallicity-density-temperature field space) than the galaxies used to derive the observational scaling relations. Second, because dust serves as a sink for metals in the ISM (as well as a source in locations that the dust may be destroyed, e.g. in the warm circumgalactic medium [CGM]), post-processing the dust content may lead to miscalculations in the total metallicity of galaxies over cosmic time (indeed, this is shown explicitly in Davé et al. (2019)). Moreover, developing a predictive model for the evolution of the cosmic dust content, dust mass functions, or dust to gas ratios requires a self-consistent treatment of dust in galaxy evolution simulations ( $\mathrm{Li}$ et al. (2019)).

In these proceedings, we describe a model for the inclusion of the formation, growth, and evolution of dust grains on-the-fly in cosmological hydrodynamic galaxy formation simulations. Much of the relevant work has been already discussed in Li et al. (2019), and these proceedings merely summarize these efforts, as well as ongoing efforts to develop a evermore sophisticated model.

\section{Relevant Physics}

In Figure 1, we summarize by schematic the relevant physical processes for dust formation, growth, and destruction. In what follows, we describe these processes in more detail. These algorithms follow work by McKinnon et al. $(2016,2018)$ and Popping et al. (2017), with some modificiations as described in Davé et al. (2019) and Li et al. (2019).

First, dust has to form. Dust is produced from the condensation of metals from both supernovae, as well as in AGB ejecta. In the formation equations, first developed by Dwek (1998), with updates by Popping et al. (2017), the mass of dust formed in each process is a fraction of the ejecta mass, modulated by a condensation efficiency, $\delta$. This efficiency is a free parameter (with ranges constrained by theoretical ejecta models, e.g. Ferrarotti \& Gail (2006); Bianchi \& Schneider (2007)), and is dependent on the chemical composition of the ejecta (i.e. the $\mathrm{C} / \mathrm{O}$ ratio).

After dust forms, it can either grow or erode. The growth processes are twofold: it can either accrete metals from surrounding gas, or collide with another dust particle (otherwise known as "coagulation") to grow in mass. The growth rate is of course dependent on the local metal mass, though is also inversely related the density, temperature and metallicity (all of which are subsumed into a characteristic accretion time scale for dust growth; see Li et al. (2019) for details). The zero-points of the density/temperature/metallicity are also free parameters. The coagulation rates are dependent on the masses of grain particles, their grain sizes, and their relative velocities.

Finally, dust can be destroyed via four processes in our model. First, electrons colliding with dust grains can erode away metals from the grain in a process known as "thermal sputtering". This is of course more efficient in ionized regions, where the time scales are controlled by the gas density and temperature. Second, dust can be destroyed in supernovae shocks. While SNe blast waves are rarely resolved in galaxy formation simulations, these can be treated in a subresolution manner, with destruction rates tied to the local type II SNe rate and local gas mass. Third, in a process known as "shattering", dust grains can smash together, and in a process analgous to coagulation, break apart. And finally in an entirely numerical effect, dust can be consumed by star formation. The latter process simply corresponds to algorithms where the dust is locked to gas particles (in particle-based codes), and the entire gas particle turns into a star particle in a star formation event. 

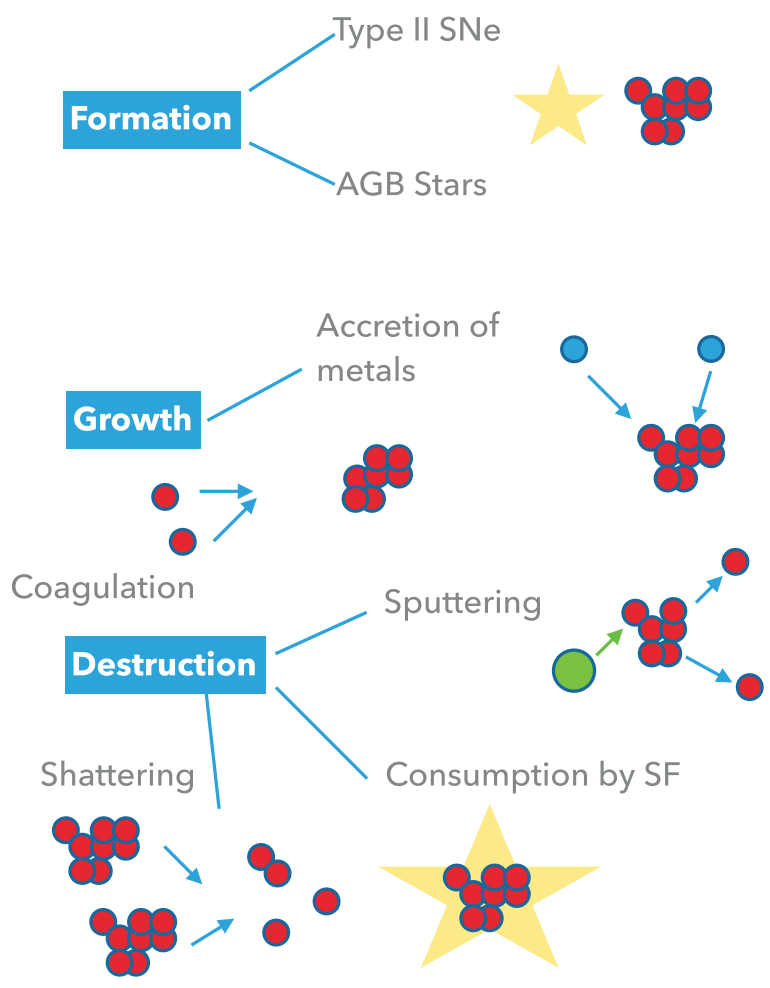

Figure 1. Schematic summarizing main physical processes of dust grains in cosmological hydrodynamic galaxy formation simulations. Dust grains form in supernovae and AGB stars (top section), and grow via the accretion of metals in the ISM, as well as coagulation (the process of sticking together). Grains are destroyed via thermal sputtering (when a hot electron collides with a grain), shattering (when grains collide with one another) or (due to numerical resolution) when they are consumed by a star-forming particle. Note, the latter process is entirely artificial, and simply stands as a proxy for other aforementioned processes in regions near forming stars.

\section{Implementation of Self-Consistent Dust Physics into Cosmological Simulations}

We have implemented the physics described in $\S 2$ and Figure 1 into the SIMBA cosmological galaxy formation model (Davé et al. (2019). In our initial implementation, we have put in a scaled-down version of the aforementioned physics for simplicity. Here, we have included just growth by accretion, and destruction by sputtering, supernovae, and star formation. In other words, in our initial implementation, we neglect coagulation and shattering. Why do we do this? The simplest model is one in which we attach the dust grains to the gas particles, and passively advect the dust alongside the gas. Here, we ideally want to minimize the number of grain sizes that we model since each bin in size adds additional memory requirements for every gas particle. So, in this simplest incarnation of the dust model, we do not model a spectrum of grain sizes, which prevents us from calculating the shattering and coagulation rates (that depend on the grain sizes). As we will discuss downstream, we are in the process of updating the algorithms to model a spectrum of grain sizes.

The primary SIMBA cosmological hydrodynamic simulation simulates a cube of $100 / h$ Mpc side length with $1024^{3}$ gas elements, and is run from $z=249$ down to redshift $z=0$. The main simulation details are described in Davé et al. (2019), and we summarize only the salient points here. The gas particle mass resolution is $1.82 \times 10^{7} M_{\odot}$, while the 


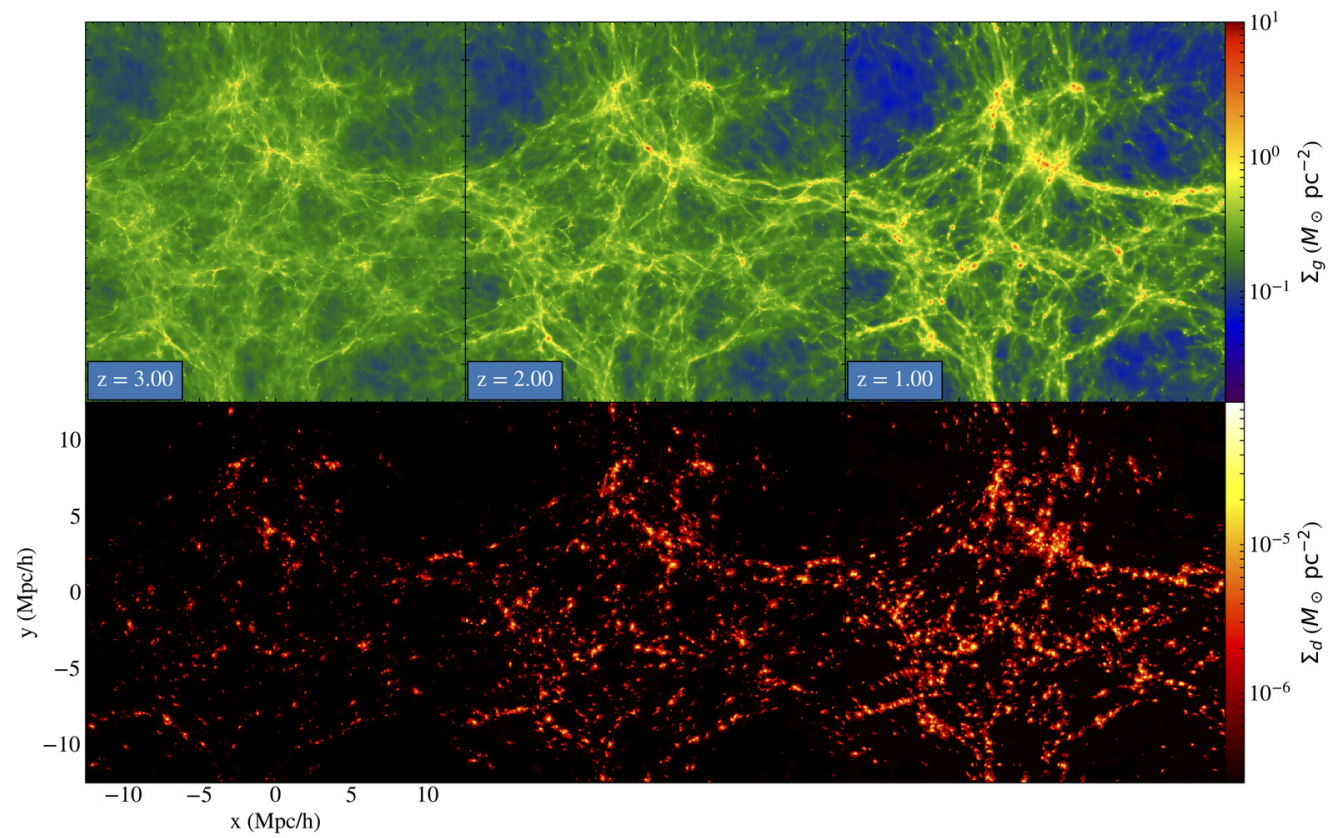

Figure 2. Gas and dust surface density projection maps of a pilot cosmological simulation at redshifts $z=1,2,3$. Dust broadly traces the overdensities in which galaxies form, though at early times is relatively low abundance due to the lack of metals.

dark matter particle resolution is $9.6 \times 10^{7} M_{\odot}$. The hydrodynamics are evolved using a forked version of the GIZMo hydrodynamics code (Hopkins (2014)) in meshless finite mass (MFM) mode.

Radiative cooling and heating from photoionization are calculated with the GRACKLE3.1 libraries (Smith et al. (2017)), in which we include metal line cooling. The neutral gas is broken into an $\mathrm{HI}$ and $\mathrm{H}_{2}$ component following the subresolution model of Krumholz et al. (2009), which balances $\mathrm{H}_{2}$ formation rates against photodissocation by LymanWerner band photons. Stars form in this molecular gas with a rate $\mathrm{SFR}=\epsilon_{*} \rho_{\mathrm{H} 2} / t_{\mathrm{dyn}}$, where $\epsilon_{*}=0.02$. These stars feed back into the interstellar medium with kinetic energies, ejection probabilities, and mass loading factors designed to mimic the global galaxy wind scaling relations derived from the very high-resolution FIRE galaxy zoom simulation series (Hopkins et al. (2018)). Finally, sIMBA includes a model for black hole accretion and feedback. These included physics help shape the physical properties of the galaxies to produce a realistic population of galaxies.

\section{Initial Results: The Dust to Gas and Dust to Metals Ratio in Galaxies}

In Figure 2, we show three $25 / \mathrm{hMpc}$ projection plots in time at redshifts $z=1,2,3$ showing the gas and dust surface density for a pilot simulation. These projections were made with the YT software (Turk et al. (2011)). Beyond serving as eye-candy (which, admittedly, is the primary concern of most galaxy formation theorists), Figure 2 shows how the large scale structure of dust in an evolving cosmological simulation grows with time.

As a first test of our model, we compare our simulated dust mass functions against observational constraints by Dunne et al. (2003); Eales et al. (2009); Dunne et al. (2011); Clemens et al. (2013) and Beeston et al. (2018). In Figure 3, we show the simulated and 


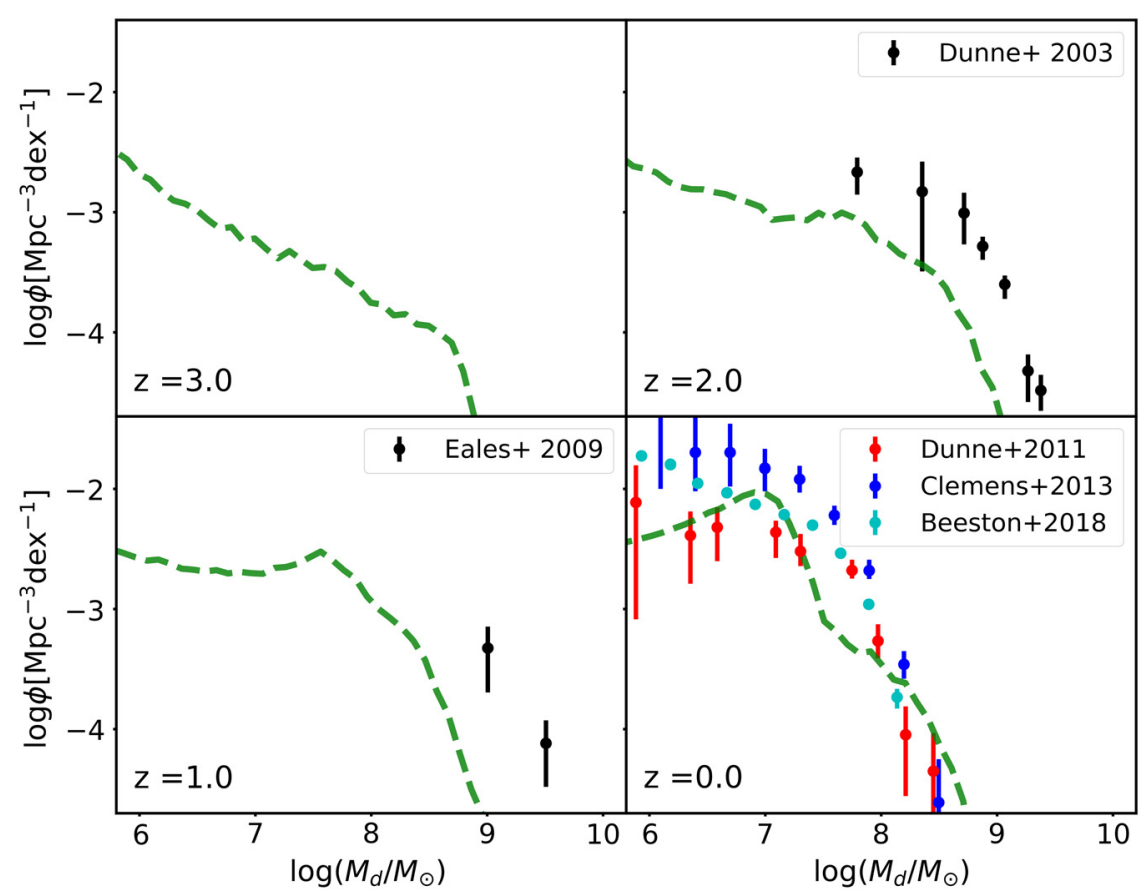

Figure 3. Model dust mass functions from the SIMBA $\left(100 / h^{3}\right)$ Mpc volume compared to observations by Dunne et al. (2003); Eales et al. (2009); Dunne et al. (2011); Clemens et al. (2013) and Beeston et al. (2018). Our model does a reasonable job at matching the locus of observed points at low redshift, though slightly underpredicts the observed dust masses at redshifts $z=1,2$. This figure first published by Li et al. (2019).

observed dust mass functions at redshifts $z=0-3$, as reproduced from Li et al. (2019). The models do a reasonable job matching the observed dust mass function at redshift $z=0$, though slightly under-predict the observed constraints at redshifts $z=1-2$. The underproduction of dust at high-redshift somewhat generic one amongst galaxy formation models that include dust (e.g. McKinnon et al. (2016); Popping et al. (2017). This may point to a number of issues, including incomplete physics in our model description of dust formation, growth and destruction, evolving parameters that we otherwise hold fixed (such as the condensation efficiencies), or issues with our understanding of stellar yields at high-redshift. Of course, as the last refuge of the scoundrel theorist, we also blame the observations: we note that the observational data points at high-redshift are scant few, and potential future observations may be in agreement with the growing number of simulations that underpredict the dust number counts at high-redshift.

We now turn from verification to prediction. What we would like to know is: "can we predict the quantity of dust a galaxy has, given a set of observed physical properties?". As a first point of investigation, we plot the dust to gas mass ratio (DGR) as a function of the galaxy gas-phase metallicity (normalized to solar) in Figure 4. Here, we color-code our simulated data based on the galaxy specific star formation rate ( $\mathrm{sSFR} \equiv \mathrm{SFR} / M_{*}$ ), and when available, show the observational constraints.

Generally, the dust to gas mass ratio decreases with metallicity, and follows a nearly constant relationship at all redshifts. The primary discriminant between high and low redshift snapshots is (a) the number of galaxies, and (b) the appearance of a red clump at late times, representing quenched galaxies with low sSFR. By and large, the models match the few observational constraints that exist at $z \approx 0$ (Rémy-Ruyer et al. (2014); 


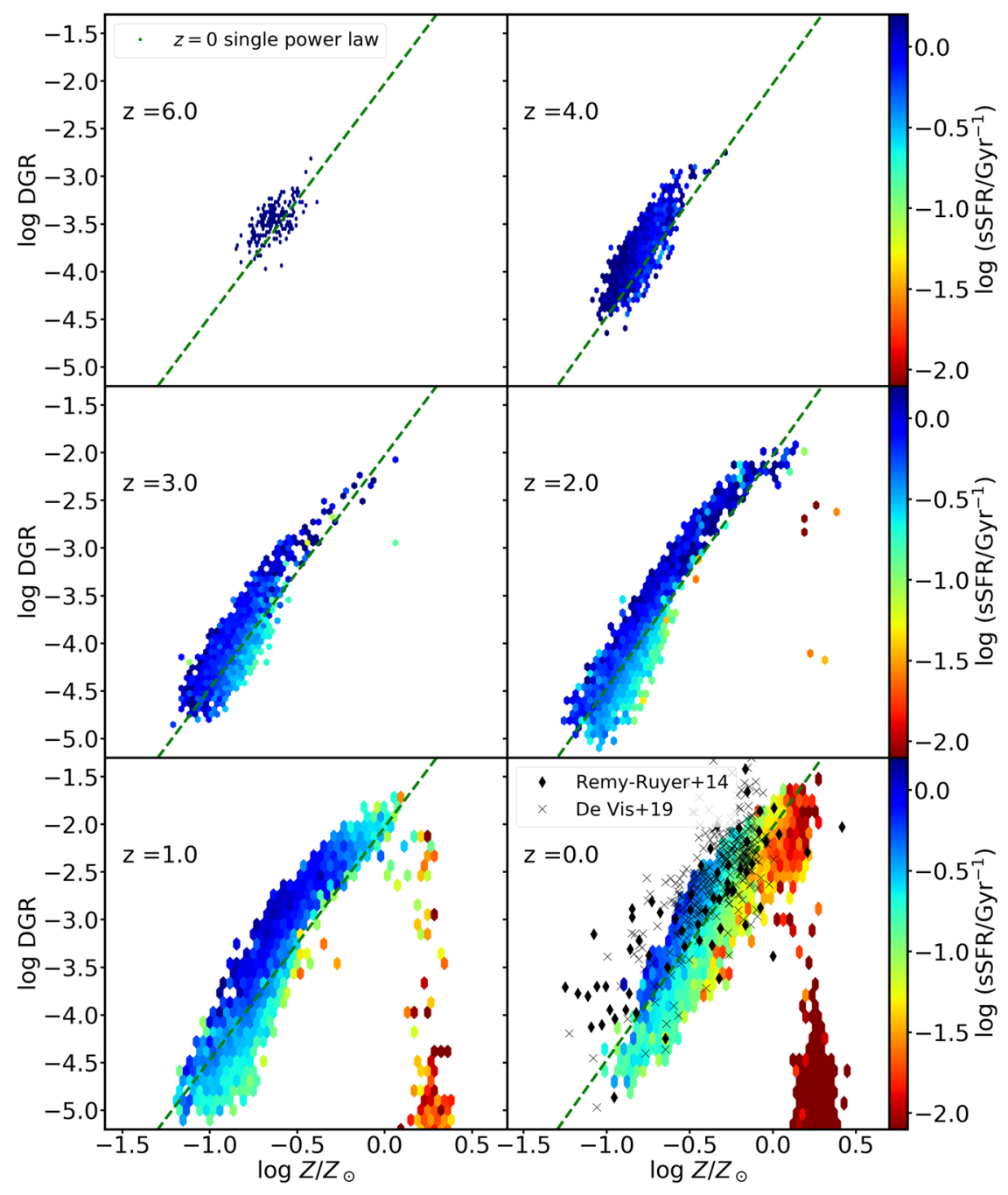

Figure 4. Dust to gas mass ratio of our model galaxies vs their gas phase metallicity. Colors show the sSFR of the galaxies, while black points show observational constraints when available (from Rémy-Ruyer et al. (2014); De Vis et al. (2019)). The dust to gas ratio for main sequence star-forming galaxies follows roughly a power-law relation with the metallicity, though as we discuss in the main text, there are secondary parameters that are non-negligible. Figure first published by Li et al. (2019).

De Vis et al. (2019)). This said, as we will discuss shortly, while a single powerlaw reasonably represents the relationship between the dust to gas ratio and metallicity, there are important secondary parameters that, when considered, can increase the accuracy of derived dust masses. We predict similar relationships for the dust to metals ratio, and show these in Li et al. (2019).

Figure 4 suggests that if one knows the gas-phase metallicity of a galaxy, within some dispersion, they can back out the galaxy dust to gas ratio. This said, the metallicity alone is not the best predictor for the dust to gas ratio. To demonstrate this, we employ a machine learning approach known as Extremely Randomized Trees (ERT) to develop a map between the dust to gas ratio and galaxy physical parameters. We search for a mapping between DGR (and the dust to metals ratio) and the metallicity, gas 
depletion time scaleback $\left(t_{\mathrm{dep}}=M_{g} / \mathrm{SFR}\right)$, stellar mass, half-baryonic mass radius, gas mass fraction $\left(f_{g}=M_{g} /\left(M_{g}+M_{*}\right)\right)$, and gas surface density $\left(\Sigma_{g}\right)$. While the details are published in $\mathrm{Li}$ et al. (2019), we find that alongside the metallicity (which, in a normalized sense, has an importance level of $\sim 0.5$ in predicting the dust to gas ratio), the gas fraction and depletion time scale combine to a nearly comparable importance. At https://bitbucket.org/lq3552/dust_galaxy_analyzer, we provide a public code base to map from physical properties of galaxies to the dust to gas and dust to metals ratios. These python codes can be used by observers and theorists to derive the dust content of their observed/modeled galaxies.

\section{Attenuation Laws and Extinction Laws}

A natural application of modeling dust in galaxies is to understand the role of attenuation and extinction in galaxies. The two concepts are different. Extinction refers to the wavelength-dependent removal of photons from the line of sight, whereas attenuation folds in the complexities of the large scale geometry of a galaxy, and therefore accounts for scattering of light back into the line of sight, as well as the contribution by unobscured stars to the UV/optical flux. The extinction curve is determined by the combination of the dust grain size distribution with the optical properties of the grains themselves (including the fractional contribution to the grain population by silicates and graphites). Attenuation curves build on this by convolving the grain properties with the complexities of galaxy geometry. For unresolved or large scale observations of galaxies, the attenuation curve is the relevant concept in understanding how much UV/optical energy was removed.

Even for a constant set of grain properties, the attenuation curve in galaxies can vary dramatically. As an example, Figure 5, we take a $(25 / h)^{3} \mathrm{Mpc}$ cosmological simulation run with the MUFASA physics suitet, and assume an fixed extinction curve for the grains in the galaxył. We infer the attenuation curves by running the dust radiative transfer package POWDERDAY (Narayanan et al. (2015), which is publicly available (https://powderday.readthedocs.io/en/latest/). We show these for snapshots at $z=0,2,4,6$. The green squares are a hexbin denoting the number of galaxies with a given curve, while the pink show the median and $1 \sigma$ standard deviation.

As is evident, even with a constant extinction curve, there is dramatic variation in the inferred attenuation curve of a galaxy due entirely to the complexities of the star-dust geometry. As we discuss in Narayanan et al. (2018), variations in the attenuation curve arise due to unobscured stars. For normalized curves, unobscured OB stars flatten (grey) attenuation curves, whereas unobscured old stars steepen normalized curves. The $2175 \AA$ bump in attenuation can be filled in due to an excess of UV flux from unobscured OB stars.

In principle, one could use this sort of study to predict the attenuation curve an observer would ideally assume for their observations. As reviewed by Walcher et al. (2011) and Conroy (2013), the dust attenuation curve is one of the more critical aspects to deriving correct physical parameters in fitting the SEDs of galaxies. One could imagine combining the attenuation curves derived from dust radiative transfer modeling with the synthetic SEDs and a machine learning algorithm to inform observers, based solely on their UV/OIR photometry, what the underlying attenuation curve of their observation is. Unfortunately, while the study of Narayanan et al. (2018) unpacked the origin of

$\dagger$ MUfASA is the parent simulation suite of SIMBA Davé et al. (2016).

$\ddagger$ These simulations were run prior to our development of the dust physics suite in the SIMBA simulations, and as such use the now antiquated method of including dust via a constant dust to metals mass ratio across all galaxies. 

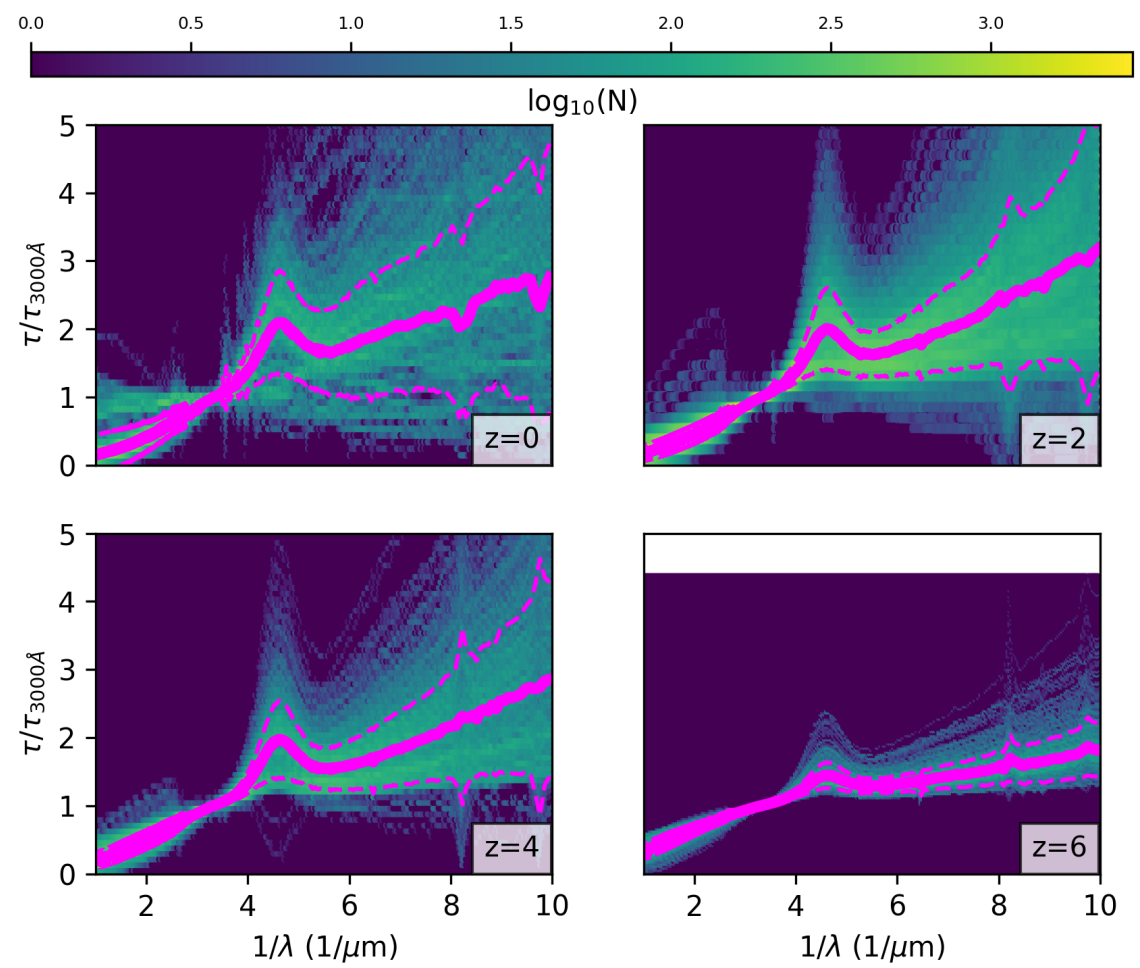

Figure 5. Attenuation curves from a $(25 / h)^{3}$ Mpc MUFASA volume with a fixed underlying extinction curve (taken originally from Narayanan et al. (2018)). Shown are panels for redshifts $z=0,2,4,6$.

variations in an attenuation curve, it is not yet predictive for what sort of attenuation curve an observer can expect for a particular galaxy. This is because Narayanan et al. (2018) employed a fixed extinction curve. The next step in deriving realistic attenuation curves for galaxies is to develop a physical model for the underlying extinction curves of galaxies. This is where dust modeling comes in.

We have recently adapted our model to include a size distribution of dust grains. To do this, we have included a new particle type into the GIZMO simulation code that represents dust, and is therefore decoupled in its dynamics from the gas (Q. Li, Narayanan \& Davé et al. in prep.). We do this following the algorithms outlined by McKinnon et al. (2018), though for the first time include these in bona fide cosmological simulations. With knowledge of the grain sizes, we include the physics of coagulation for grain growth, and shattering for destruction. The grains are treated as spherical objects with mass $m(a)=4 / 3 \pi \rho_{\text {grain }} a^{3}$, where $\rho_{\text {grain }}$ is the dust grain density, and $a$ is the size. We assume a fixed grain density of $\rho_{\text {grain }}=2.4 \mathrm{~g} \mathrm{~cm}^{-3}$ as reviewed by Draine (2003), and therefore infer the size, $a$ from the grain mass. The box sizes for the active dust are necessarily smaller than the original SIMBA volumes due to the need for extremely high resolution to faithfully model coagulation and shattering processes. The initialized size distributions are assumed to follow the theoretical calculations of Asano et al. (2013) for the AGB ejecta-produced grains, and Nozawa et al. (2007) for the SNe produced grains. In Figure 6, we show the modeled extinction curves at $z=0$ of $\sim 50$ galaxies of a pilot $(6 / h)^{3} \mathrm{Mpc}$ box with full SIMBA physics, and the aforementioned updated active dust model. We overplot the curve expected for a Mathis et al. (1977) distribution for the Galaxy in red. As is evident, a diverse range of extinction curves exist for local galaxies in this small 


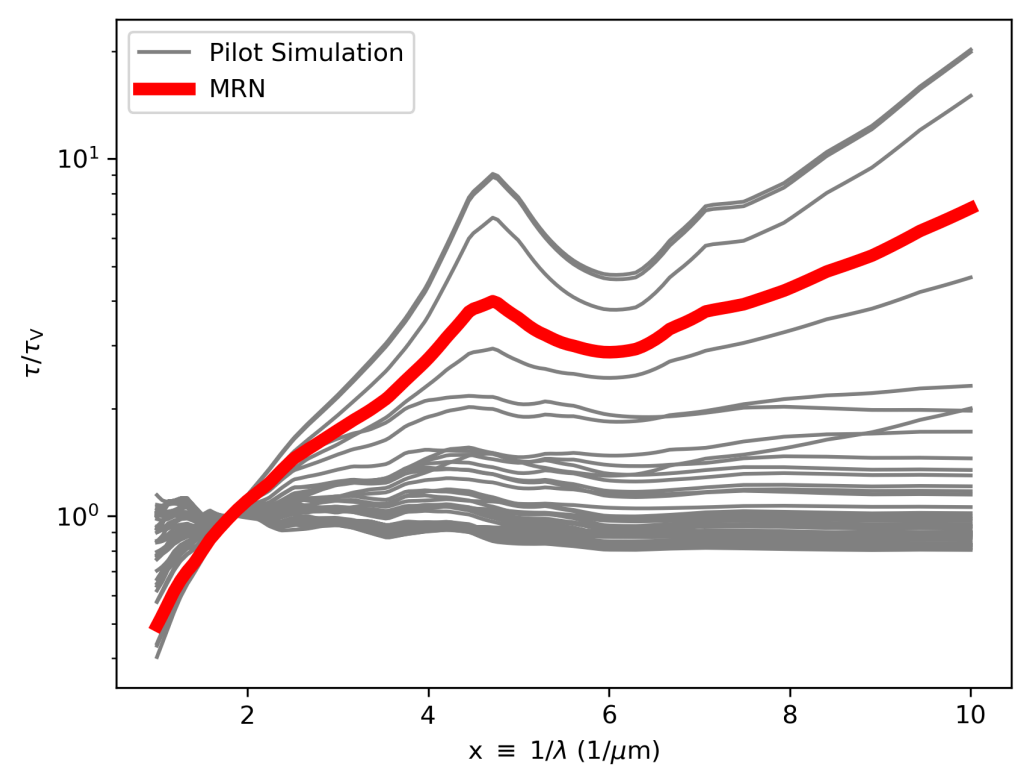

Figure 6. Model extinction curves for a pilot small box $\left((6 / h)^{3} \mathrm{Mpc}\right)$ SIMBA volume, with a model for active dust grains with a spectrum of sizes. The model output is the final dust size distribution for each galaxy at $z=0$ which, when convolved with an assumed set of optical properties for the galaxy, results in a model extinction curve.

box, though at least a handful have properties similar to the Milky Way's. We note that these results are a pilot study, and meant to be taken more as a proof of concept, rather than conclusive.

\section{Relationship to other Models}

A number of groups world-wide have published or are developing complementary models for including the physics of dust formation/growth/destruction in galaxy formation models, highlighting the importance of this physics in both understanding galaxy evolution, but also comparing models to observations across the electromagnetic spectrum.

McKinnon et al. (2016); Hou et al. (2017); Popping et al. (2017); Aoyama et al. (2018); Hou et al. (2019); Vijayan et al. (2019) and Vogelsberger et al. (2019) have all included similar dust physics in cosmological hydrodynamic simulations, or semi-analytic models. As previously mentioned, no model is able to match the $z=2$ dust mass function to date, though our presented model appears to be the closest match, underpredicting by a factor $\sim 3$. These works model a single grain size, and therefore generally focus on global scaling relations, as in our work (Li et al. (2019).

More recently, a number of groups have turned their attention to simulating a spectrum of grain sizes. Hou et al. $(2017,2019)$ employ a 2-size approximation, binning grains as "small" or "large", with $a=0.03 \mu \mathrm{m}$ as the dividing line (Hirashita (2015)). While an approximation to a more finely binned spectrum of sizes, Hirashita (2015) have shown that in some test cases the two-size approximation can reproduce the results of more finely binned simulations. McKinnon et al. (2018) has developed a model for a full spectrum of grain sizes (that we adopt here in generating Figure 6) that has been applied to idealized disk galaxies with the AREPO hydrodynamic code. 


\section{Looking Forward}

The relatively new field of including the physics of dust formation, growth, and destruction on the fly in galaxy formation simulations is a promising one. There are several important steps forward to take with these simulations:

- Galaxy formation models should begin to include the physics of dust cooling when modeling the low temperature cooling in simulations.

- A new generation of ultra high-resolution cosmological simulations, or zoom simulations will elucidate our understanding of the origin of variations in extinction laws, and the origin of the $2175 \AA$ bump in galaxies.

- In the long run, combining the results of these sorts of simulations with SED fitting software will enable a modern approach to accurately deriving the physical properties of galaxies from observed photometry.

- Not yet mentioned in this proceedings, though prevalent in both the community as well as this wonderful conference is the topic of the origin of high-redshift dusty galaxies. Simulations that include self-consistent models for dust physics will begin to address, for the first time, the origin of the highest-redshift dusty galaxies (e.g. Casey et al. (2014); Marrone et al. (2018)). The initial comparisons to redshift $z=2$ dust mass functions of modern simulations may indicate a different formulation of the age-old tension between submillimeter-selected galaxies at high-redshift, and observations (e.g. Narayanan et al. $(2010))$.

\section{Acknowledgements}

The authors thank the organizers of this meeting for a lively and educational conference in a phenomenal location, Viana do Castelo, Portugal. This research made use of the YT code base, and the authors are grateful to the community developers of this software. D. N. would like to extend gratitude to Cristina Fernandes and Gabriel Menezes for taking him surfing on the first day of the meeting, and appreciates the organizers patience while he skipped the panel he was otherwise slated to participate in, to go surf.

\section{References}

Abruzzo, M. W., Narayanan, D., Davé, R., et al. 2018, arXiv e-prints, arXiv:1803.02374

Asano, R. S., Takeuchi, T. T., Hirashita, H., et al. 2013, MNRAS, 432, 637

Aoyama, S., Hou, K.-C., Hirashita, H., et al. 2018, MNRAS, 478, 4905

Beeston, R. A., Wright, A. H., Maddox, S., et al. 2018, MNRAS, 479, 1077

Bianchi, S. \& Schneider, R. 2007, MNRAS, 378, 973

Casey, C. M., Narayanan, D., \& Cooray, A. 2014, PhR, 541, 45

Clemens, M. S., Negrello, M., De Zotti, G., et al. 2013, MNRAS, 433, 695

Conroy, C. 2013, ARA\&A, 51, 393

Davé, R., Thompson, R., \& Hopkins, P. F. 2016, MNRAS, 462, 3265

Davé, R., Anglés-Alcázar, D., Narayanan, D., et al. 2019, MNRAS, 486, 2827

De Vis, P., Jones, A., Viaene, S., et al. 2019, A\&A, 623, A5

Draine, B. T. 2003, ApJ, 598, 1017

Draine, B. T. 2011, Physics of the Interstellar and Intergalactic Medium by Bruce T. Draine. Princeton University Press

Dunne, L., Eales, S. A., \& Edmunds, M. G. 2003, MNRAS, 341, 589

Dunne, L., Gomez, H. L., da Cunha, E., et al. 2011, MNRAS, 417, 1510

Dwek, E. 1998, ApJ, 501, 643

Eales, S., Chapin, E. L., Devlin, M. J., et al. 2009, ApJ, 707, 1779

Ferrarotti, A. S. \& Gail, H.-P. 2006, A\&\&A, 447, 553

Goldsmith, P. F. 2001, ApJ, 557, 736

Hirashita, H. 2015, MNRAS, 447, 2937

Hou, K.-C., Hirashita, H., Nagamine, K., et al. 2017, MNRAS, 469, 870 
Hou, K.-C., Aoyama, S., Hirashita, H., et al. 2019, MNRAS, 485, 1727

Hopkins, P. F., Wetzel, A., Kereš, D., et al. 2018, MNRAS, 480, 800

Krumholz, M. R., McKee, C. F., \& Tumlinson, J. 2009, ApJ, 699, 850

Hayward, C. C., Narayanan, D., Kereš, D., et al. 2013, MNRAS, 428, 2529

Hopkins, P. F. 2014, GIZMO: Multi-method magneto-hydrodynamics+gravity code, ascl:1410. 003

Lagos, C. del P., Robotham, A. S. G., Trayford, J. W., et al. 2019, arXiv e-prints, arXiv:1908.03423

Li, Q., Narayanan, D., \& Davé, R. 2019, arXiv e-prints, arXiv:1906.09277

Marrone, D. P., Spilker, J. S., Hayward, C. C., et al. 2018, Nature, 553, 51

Mathis, J. S., Rumpl, W., \& Nordsieck, K. H. 1977, ApJ, 217, 425

McKinnon, R., Torrey, P., \& Vogelsberger, M. 2016, MNRAS, 457, 3775

McKinnon, R., Vogelsberger, M., Torrey, P., et al. 2018, MNRAS, 478, 2851

Narayanan, D., Hayward, C. C., Cox, T. J., et al. 2010, MNRAS, 401, 1613

Narayanan, D., Turk, M., Feldmann, R., et al. 2015, Nature, 525, 496

Narayanan, D. \& Krumholz, M. R. 2017, MNRAS, 467, 50

Narayanan, D., Conroy, C., Davé, R., et al. 2018, ApJ, 869, 70

Nozawa, T., Kozasa, T., Habe, A., et al. 2007, ApJ, 666, 955

Popping, G., Somerville, R. S., \& Galametz, M. 2017, MNRAS, 471, 3152

Privon, G. C., Narayanan, D., \& Davé, R. 2018, ApJ, 867, 102

Rémy-Ruyer, A., Madden, S. C., Galliano, F., et al. 2014, Aø̛A, 563, A31

Smith, B. D., Bryan, G. L., Glover, S. C. O., et al. 2017, MNRAS, 466, 2217

Turk, M. J., Smith, B. D., Oishi, J. S., et al. 2011, ApJS, 192, 9

Vijayan, A. P., Clay, S. J., Thomas, P. A., et al. 2019, arXiv:1904.02196

Vogelsberger, M., McKinnon, R., O'Neil, S., et al. 2019, MNRAS, 487, 4870

Watson, D. 2011, A\& A, 533, A16

Walcher, J., Groves, B., Budavári, T., et al. 2011, ApESSS, 331, 1 